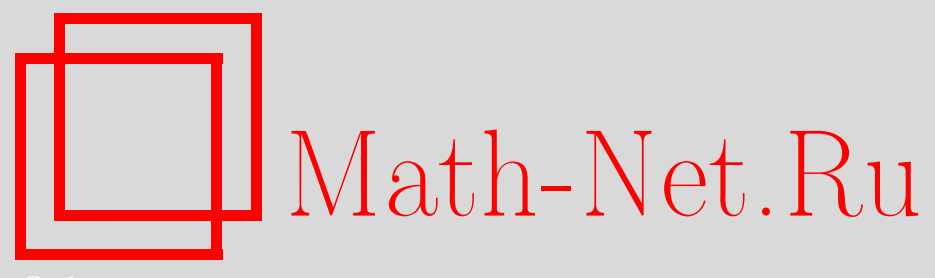

А. М. Зубков, Вычисление распределений характеристик чисел компонент и циклических точек случайного отображения, Mатем. вопр. криптогр., 2010, том 1, выпуск 2, 5-18

DOI: https://doi.org/10.4213/mvk7

Использование Общероссийского математического портала Math-Net.Ru подразумевает, что вы прочитали и согласны с пользовательским соглашением http://www.mathnet.ru/rus/agreement

Параметры загрузки:

IP : 3.85 .5 .30

26 апреля 2023 г., 14:26:03

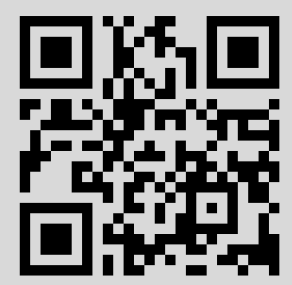




\author{
МАТЕМАТИЧЕСКИЕ ВОПРОСЫ КРИПТОГРАФИИ \\ 2010 T. 1 № 2 C. 5-18
}

УДК: 519.212 .2

\title{
Вычисление распределений чисел компонент и циклических точек случайного отображения
}

\author{
А. М.Зубков \\ Математический институт им. В. А. Стеклова РАН, Москва
}

Получено 22.IV.2010

В работе описаны использующие аппарат цепей Маркова алгоритмы точного вычисления распределений чисел компонент и циклических точек случайного отображения конечного множества в себя и итерации двух таких независимых отображений.

Ключевые слова: случайные отображения, циклические точки, вычисление распределений

Computation of distributions of the numbers of components and cyclic points for random mappings

\section{A. M.Zubkov}

Steklov Mathematical Institute of RAS, Moscow

Abstract. Markov chain based algorithms for the exact computation of distributions of the numbers of components and cyclic points for the random mapping of a finite set into itself and for the iteration of two such mappings are described.

Key words: random mapping, cyclic points, computation of distributions

Citation: Mathematical Aspects of Cryptography, 2010, vol. 1, no. 2, pp. 5-18 (Russian). 
Случайные отображения конечных множеств изучались в ряде работ (см., например, [1-5] и приведенную в них библиографию). Как правило, результаты исследования различных свойств случайных отображений формулируются в виде предельных теорем, однако утверждения предельных теорем не позволяют в конкретных случаях получать численные результаты, поскольку оценки скорости сходимости в этих теоремах либо отсутствуют, либо оказываются довольно грубыми. Кроме того, предельные теоремы, как правило, не дают информации о значениях вероятностей в области больших уклонений. Основная масса предельных теорем относится к равновероятным отображениям.

Цель настоящей работы - указать способ точного вычисления некоторых характеристик случайных отображений, в частности, распределения числа связных компонент и числа циклических точек.

Пусть $\varphi-$ случайное равновероятное отображение конечного множества $S=\{1, \ldots, N\}$, т. е. значения $\varphi(1), \ldots, \varphi(N)$ независимы и имеют равномерное распределение на $S$. Случайному отображению $\varphi$ соответствует случайный ориентированный граф $G=G(\varphi)$ с множеством вершин $S$ и множеством ориентированных ребер $(k, \varphi(k)), k \in S$. Обозначим через $\nu_{N}=\nu(\varphi)$ число связных компонент графа $G$. Известно (см. [1]), что для любого $x, 0<x \leq e^{-1}$, и любого $n$

$$
\boldsymbol{P}\left\{\nu_{N}=n\right\}=\frac{N ! \ln ^{n} A(x)}{n ! N^{N} x^{N}} \boldsymbol{P}\left\{\xi_{1}+\ldots+\xi_{n}=N\right\},
$$

где $A(x)=\sum_{k=0}^{\infty} \frac{k^{k} x^{k}}{k !}$, случайные величины $\xi_{1}, \ldots, \xi_{n}$ независимы, одинаково распределены и

$$
\boldsymbol{P}\left\{\xi_{1}=k\right\}=\frac{b_{k} x^{k}}{k ! B(x)}, \quad k=1,2, \ldots
$$

a

$$
B(x)=\sum_{k=1}^{\infty} \frac{b_{k} x^{k}}{k !}=\ln A(x), \text { т. е. } b_{k}=(k-1) ! \sum_{m=0}^{k-1} \frac{k^{m}}{m !} .
$$

Из этих соотношений в [1] выводятся асимптотические формулы

$$
\boldsymbol{P}\left\{\nu_{N}=n\right\}=\frac{1}{n !}\left(\frac{1}{2} \ln N\right)^{n} e^{-\frac{1}{2} \ln N}(1+o(1))
$$

и

$$
\boldsymbol{P}\left\{\nu_{N}=n\right\}=\frac{1+o(1)}{\sqrt{\pi \ln N}} \exp \left\{-\frac{\left(n-\frac{1}{2} \ln N\right)^{2}}{\ln N}\right\},
$$


которые при $N \rightarrow \infty$ выполняются равномерно относительно целых $n$, для которых $\left(n-\frac{1}{2} \ln N\right)(\ln N)^{-7 / 12}$ лежит в любом конечном интервале.

Точную формулу (1) можно преобразовать к виду, удобному для вычисления распределения числа компонент $\nu_{N}$ при значениях $N$, допускающих размещение нескольких массивов по $N$ чисел в памяти ЭВМ:

$$
\boldsymbol{P}\left\{\nu_{N}=n\right\}=\frac{N !}{n ! N^{N}} \operatorname{coef}_{x^{N}} D_{N}^{n}(x), \quad D_{N}(x)=\sum_{k=1}^{N} \frac{x^{k}}{k} \sum_{m=0}^{k-1} \frac{k^{m}}{m !} .
$$

Вычисление $\operatorname{coef}_{x^{k}} D_{N}(x)$ можно провести за $O(k)$ арифметических операций; вычисление всех $N$ коэффициентов этого многочлена - за $O\left(N^{2}\right)$ операций. Далее вычисление первых $N$ коэффициентов произведения двух многочленов степени $N$ проводится за $O\left(N^{2}\right)$ операций, поэтому вероятности $\boldsymbol{P}\left\{\nu_{N}=n\right\}$ можно вычислять последовательно по $n=1,2, \ldots$, затрачивая на вычисление каждой следующей вероятности $O\left(N^{2}\right)$ операций. Таким образом, вычисление полного распределения $\nu_{N}$ можно провести за $O\left(N^{3}\right)$ арифметических операций, а первых $M$ вероятностей - за $O\left(M N^{2}\right)$ операций.

Эта формула применима только к классической модели случайного отображения конечного множества $S$, в которой образы всех элементов независимы и имеют равномерные распределения на $S$. Однако другие модели случайных отображений тоже могут представлять интерес как с теоретической, так и с прикладной точек зрения, и распределения чисел компонент таких отображений могут отличаться от соответствующих распределений для равновероятных отображений. Примерами являются: а) отображения, являющиеся итерациями нескольких равновероятных отображений; б) отображения, в которых распределение образов не является равномерным; в) отображения, в которых распределение образа элемента зависит от этого элемента (например, случайные двудольные отображения), и т. п.

В основе предлагаемого подхода лежит использование вспомогательной цепи Маркова, которая представляет процесс построения случайного отображения (последовательного определения образов элементов) в виде, обеспечивающем монотонное неубывание числа компонент. Чтобы сделать изложение более понятным, рассмотрим сначала случай равновероятных отображений.

Пусть требуется найти число $\nu_{N}$ связных компонент отображения $\varphi: S=\{1, \ldots, N\} \rightarrow S$. Чтобы задать отображение, нужно определить его значения на всех элементах множества $S$, и эти элементы можно перебирать в любом порядке. 
Рассмотрим процесс $\mathcal{P}$ перебора элементов множества $S$, состоящий из отдельных шагов. Состоянием процесса в начале $(t+1)$-го шага, $t=$ $=0,1, \ldots$, является пара $(\sigma(t), \nu(t))$, где $\sigma(t) \subset S-$ множество всех элементов $x \in S$, для которых значение $\varphi(x)$ было определено на каком-нибудь из первых $t$ шагов, а $\nu(t)$ - число связных компонент графа отображения $\varphi$, ограниченного на $\sigma(t)$, т. е. графа с множеством вершин $\sigma(t)$ и множеством peбер $(x, \varphi(x)), x \in \sigma(t)$. В частности, $(\sigma(0), \nu(0))=(\varnothing, 0)$.

Шаг с номером $t=1,2, \ldots$ начинается с выбора произвольного элемента $x$ множества $S \backslash \sigma(t-1)$; на нем значение отображения $\varphi$ еще не определено. После этого находим значения $\varphi(x), \varphi^{2}(x), \varphi^{3}(x), \ldots$ до тех пор, пока не появится значение $\varphi^{\tau(t)}(x) \in \sigma(t-1) \cup\left\{x, \varphi(x), \ldots, \varphi^{\tau(t)-1}(x)\right\}$, на котором к этому моменту отображение $\varphi$ уже определено. Тогда $t$-й шаг заканчивается. Возможны два варианта: либо $\varphi^{\tau(t)}(x) \in \sigma(t-1)$, либо $\varphi^{\tau(t)}(x) \in$ $\in\left\{x, \varphi(x), \ldots, \varphi^{\tau(t)-1}(x)\right\}$. В первом случае все элементы построенной цепочки присоединяются к одной из ранее образовавшихся связных компонент отображения $\varphi$, во втором случае они порождают новую связную компоненту. Таким образом, в конце каждого шага процесса $\mathcal{P}$ число связных компонент либо не изменяется, либо увеличивается на 1 . Когда все элементы множества $S$ будут исчерпаны, число связных компонент определится полностью.

Если $\varphi-$ случайное отображение, то процесс $\mathcal{P}=\{(\sigma(t), \nu(t))\}_{t \geq 0}$ тоже является случайным с множеством состояний $2^{S} \times\{0,1, \ldots, N\}$; здесь $2^{S}$ - совокупность всех подмножеств множества $S$. Начальным состоянием процесса является $\{(\varnothing, 0)\}$; состояния $(s, n) \in 2^{S} \times\{0,1, \ldots, N\}$ c $s=S$ - поглощающие. Финальное распределение процесса $\mathcal{P}$ на множестве $\{S\} \times\{1, \ldots, N\}$ соответствует распределению числа компонент отображения $\varphi$. Если случайное отображение $\varphi$ таково, что образы $\varphi(x), x \in S$, элементов независимы, и при любом $t=0,1, \ldots$ на $(t+1)$-м шаге начальный элемент $x \in S \backslash \sigma(t)$ выбирается независимо от значений отображения $\varphi$ на множестве $\sigma(t)$, то при фиксированном $\sigma(t)$ распределение последовательности $\{(\sigma(s), \nu(s))\}_{s>t}$ зависит только от $\sigma(t)$ и не зависит от предыстории $\{(\sigma(s), \nu(s))\}_{0 \leq s<t}$; иными словами, для отображения с независимыми образами элементов процесс $\mathcal{P}$ является марковским.

В случайном равновероятном отображении $\varphi$ образы элементов множества $S$ не только независимы, но и равномерно распределены на $S$. Поэтому марковский процесс $\mathcal{P}$ допускает укрупнение состояний: последовательность $\{(|\sigma(t)|, \nu(t))\}_{t \geq 0}$ является цепью Маркова с дискретным временем и множеством состояний $\{0,1, \ldots, N\} \times\{0,1, \ldots, N\}$. Число $(N+1)^{2}$ состояний укрупненной цепи Маркова существенно меньше, чем число $(N+1) 2^{N}$ состояний исходной цепи, и это позволяет находить ее финальное распреде- 
ление, последовательно вычисляя распределения $\{(|\sigma(t)|, \nu(t))\}, t=0,1, \ldots$, с помощью матрицы переходных вероятностей.

Выведем формулы для переходных вероятностей укрупненной цепи. Из состояния $(|\sigma(t)|, \nu(t))=(m, v)$ укрупненная цепь может перейти только в состояния $(m+u, v)$ или $(m+u, v+1)$, где $u \in\{1,2, \ldots, N-m\}$. Переход

$$
(m, v) \rightarrow(m+u, v)
$$

происходит, когда $|\sigma(t)|=m$, элементы $x, \varphi(x), \ldots, \varphi^{u-1}(x) \notin \sigma(t)$ попарно различны и $\varphi^{u}(x) \in \sigma(t)$, а переход

$$
(m, v) \rightarrow(m+u, v+1)
$$

происходит, когда $|\sigma(t)|=m$, элементы $x, \varphi(x), \ldots, \varphi^{u-1}(x) \notin \sigma(t)$ попарно различны и $\varphi^{u}(x) \in\left\{x, \varphi(x), \ldots, \varphi^{u-1}(x)\right\}$. Отсюда следует, что вероятности переходов имеют следующий вид:

$$
\begin{gathered}
p_{(m, v),(m+u, v)}=\frac{m}{N} q(m, u), \quad p_{(m, v),(m+u, v+1)}=\frac{u}{N} q(m, u), \\
q(m, u)=\prod_{k=1}^{u-1}\left(1-\frac{m+k}{N}\right) .
\end{gathered}
$$

Здесь $q(m, u)$ - вероятность события $x, \varphi(x), \ldots, \varphi^{u-1}(x) \notin\{1, \ldots, m\}$. Значения $p_{(m, v),(m+u, v)}, p_{(m, v),(m+u, v+1)}$ можно вычислить заранее. Множество состояний укрупненной цепи состоит из $O\left(N^{2}\right)$ элементов; ввиду того, что в каждой строке матрицы вероятностей переходов отличны от 0 не более $2 N$ элементов, вычисление изменения распределения цепи за один шаг можно провести за $O\left(N^{3}\right)$ арифметических операций, а если объединить все состояния с $v>M$ в одно поглощающее состояние - то за $O\left(M N^{2}\right)$ операций. Максимальное число шагов укрупненной цепи до попадания в множество финальных состояний не превышает $N$. Таким образом, без дополнительных упрощений (например, отбрасывания слагаемых, величина которых меньше допустимой погрешности) вычислительная сложность описанного метода почти в $N$ раз больше вычислительной сложности точной формулы.

Результаты вычислений распределений $\nu_{N}$ по формулам (5) для $N=$ $=100,250,500,1000,2000$ приведены в Таблице 1 и выделены жирным шрифтом. В каждой клеточке этой таблицы приводятся также значения, вычисленные по главным частям асимптотических формул (2) и (3) для пуассоновской и нормальной аппроксимаций соответственно. Эти результаты показывают, что для рассмотренных значений $N$ предельные теоремы дают не очень хорошие аппроксимации в центральной части распределений. 
А. М. Зубков

Таблица 1. Точные распределения $\boldsymbol{P}\left\{\nu_{N}=v\right\}$ чисел компонент равновероятных отображений и их пуассоновские и нормальные аппроксимации

\begin{tabular}{|c|c|c|c|c|c|}
\hline v & $N=100$ & $N=250$ & $N=500$ & $N=1000$ & $N=2000$ \\
\hline 1 & 0.1220996 & 0.0779592 & 0.0553925 & 0.0393032 & 0.0278594 \\
\hline Pois & .2302585 & 0.1746039 & 0.1389629 & 0.1092212 & 0.0849807 \\
\hline Normal & 0.1818825 & 0.1369468 & 0.1107612 & 0.0897802 & 0.0729277 \\
\hline 2 & 0.2750239 & 0.2110974 & 0.1691089 & 0.1335704 & 0.1043140 \\
\hline Pois & 0.2650949 & 0.2410172 & 0.2158999 & 0.1886183 & 0.1614825 \\
\hline Normal & 0.2577318 & 0.2162118 & 0.1857948 & 0.1580751 & 0.1335904 \\
\hline 3 & 0.2874088 & 0.2708997 & 0.2473149 & 0.2191700 & 0.1897055 \\
\hline Pois & 4679 & 0.2217945 & 0.2236222 & 0.2171549 & 0.2045687 \\
\hline Normal & 0.2365556 & 0.2376267 & 0.2258987 & 0.2083556 & 0.1880977 \\
\hline 4 & 0.187 & 0.2212013 & 0.2322667 & 0.2325047 & 0.2241839 \\
\hline Pois & 255 & 1530787 & 0.1737156 & 75066 & 0.1943634 \\
\hline Normal & 0.1406328 & 0.1818024 & 0.1990803 & 0.2055917 & 0.2035717 \\
\hline 5 & 0.8666421 & 0.1299575 & 0.1582343 & 0.1799854 & 0.1941881 \\
\hline Pois & 0.05 & 218 & 0.107 & 0.1295250 & 0.1477337 \\
\hline Normal & 0.054 & 0.0968262 & 0.127 & 8676 & 0.1693468 \\
\hline 6 & 0.0303775 & 0.0588295 & 0.0836767 & 0.1087305 & 0.1317913 \\
\hline Pois & 0.020 & 8903 & 0.0559094 & 0.0745606 & 0.0935758 \\
\hline Normal & 0.013 & 0.035 & 0.0588790 & 0.0839814 & 0.1082834 \\
\hline 7 & 0.008 & 4419 & 0.0358706 & 0.0535076 & $\mathbf{0 . 0 7 3 1 2 7 7}$ \\
\hline Pois & 0.006 & 3380 & 8182 & 0.0367890 & 0.0508043 \\
\hline Normal & 0.0021821 & 0.0092650 & 0.0197596 & 0.0347665 & 0.0532197 \\
\hline 8 & 0.00 & 0.0064886 & 0.0128485 & 0.0221014 & 0.0341721 \\
\hline Pois & 598 & 930 & 0.009 & 0.0158831 & 0.0241349 \\
\hline Normal & 0.000 & 0.0016646 & 0.0048065 & 0.0107745 & 0.0201052 \\
\hline 9 & 0.0003678 & 0.0016677 & 0.0039324 & 0.0078335 & 0.013745 \\
\hline Pois & 0.000 & 0.0016236 & 0.0033282 & 0.0060954 & 0.0101915 \\
\hline Normal & 0.000 & 0.0002082 & 0.0008475 & 0.0024997 & 0.0058381 \\
\hline 10 & 0.000 & 3705 & 0.0010463 & 0.0024232 & 0.0048394 \\
\hline Pois & 0.00011 & & & 21053 & 0.0038732 \\
\hline Normal & 0.0000007 & 0.0000181 & 0.0001083 & 0.0004342 & 0.0013030 \\
\hline 11 & 0.0000085 & 0.0000722 & 0.0002453 & 0.0006632 & 0.0015115 \\
\hline Pois & & & & 6610 & 0.0013382 \\
\hline Normal & 0.00000002 & 0.0000011 & 0.0000100 & 0.0000564 & 0.0002235 \\
\hline 12 & 0.0000010 & 0.000 & 0.0000513 & 0.0001623 & 0.0004234 \\
\hline Pois & 46 & 0259 & 0.0000756 & 0.0001903 & 0.0004238 \\
\hline Normal & $<10^{-9}$ & 0.00000005 & 0.0000007 & 0.0000055 & 0.0000295 \\
\hline
\end{tabular}


Преимуществом описанного подхода, использующего цепи Маркова, является возможность его применения к более сложным моделям случайных отображений.

Например, пусть случайное отображение $\widetilde{\varphi}: S \rightarrow S$ является итерацией двух независимых случайных отображений $\varphi_{1}$ и $\varphi_{2}$, имеющих равномерное распределение на множестве всех отображений $S \rightarrow S$, а именно: $\widetilde{\varphi}(x)=\varphi_{2}\left(\varphi_{1}(x)\right)$. По отображению $\widetilde{\varphi}$ можно построить процесс $\mathcal{P}^{(2)}$, аналогичный процессу $\mathcal{P}$ и содержащий информацию о множествах, на которых уже определены значения отображений $\varphi_{1}$ и $\varphi_{2}$.

Состоянием процесса в начале $(t+1)$-го шага, $t=0,1, \ldots$, является тройка $\left(\sigma_{1}(t), \sigma_{2}(t), \nu(t)\right)$, где $\sigma_{j}(t) \subset S-$ множество всех элементов $x \in S$, для которых значение $\varphi_{j}(x), j=1,2$, было определено на каком-нибудь из первых $t$ шагов, а $\nu(t)$ - число связных компонент графа отображения $\widetilde{\varphi}$, ограниченного на $\sigma_{1}(t)$, т. е. графа с множеством вершин $\sigma_{1}(t)$ и множеством peбер $(x, \widetilde{\varphi}(x)), x \in \sigma_{1}(t)$. В частности, $\left(\sigma_{1}(0), \sigma_{2}(0), \nu(0)\right)=(\varnothing, \varnothing, 0)$.

Шаг с номером $t=1,2, \ldots$ начинается с выбора произвольного элемента $x$ множества $S \backslash \sigma_{1}(t-1)$; на нем значения отображений $\varphi_{1}, \widetilde{\varphi}$ еще не определены. После этого находим значения $\varphi_{1}(x), \varphi_{2}\left(\varphi_{1}(x)\right)=\widetilde{\varphi}(x)$, $\varphi_{1}(\widetilde{\varphi}(x)), \varphi_{2}\left(\varphi_{1}(\widetilde{\varphi}(x))\right)=\widetilde{\varphi}^{2}(x), \ldots$ до тех пор, пока не появится значение $\tilde{\varphi}^{\tau(t)}(x) \in \sigma_{1}(t-1) \cup\left\{x, \widetilde{\varphi}(x), \ldots, \widetilde{\varphi}^{\tau(t)-1}(x)\right\}$, на котором к этому моменту отображения $\varphi_{1}$ и $\widetilde{\varphi}$ уже определены. Тогда $t$-й шаг заканчивается. Возможны два варианта: либо $\widetilde{\varphi}^{\tau(t)}(x) \in \sigma_{1}(t-1)$, либо $\widetilde{\varphi}^{\tau(t)}(x) \in$ $\in\left\{x, \tilde{\varphi}(x), \ldots, \tilde{\varphi}^{\tau(t)-1}(x)\right\}$. В первом случае все элементы построенной цепочки присоединяются к одной из ранее образовавшихся связных компонент отображения $\widetilde{\varphi}$, во втором они порождают новую связную компоненту. Таким образом, в конце каждого шага процесса $\mathcal{P}^{(2)}$ число связных компонент либо не изменяется, либо увеличивается на 1. Когда все элементы множества $S$ будут исчерпаны, число связных компонент определится полностью.

События, приводящие к указанным двум вариантам, отличаются от событий в процессе $\mathcal{P}$. Первый вариант $\left(\widetilde{\varphi}^{\tau(t)}(x) \in \sigma_{1}(t-1)\right)$ реализуется, если либо $\varphi_{1}\left(\widetilde{\varphi}^{\tau(t)-1}(x)\right) \in \sigma_{2}(t-1)$, либо $\varphi_{1}\left(\widetilde{\varphi}^{\tau(t)-1}(x)\right) \notin \sigma_{2}(t-1)$, но $\varphi_{2}\left(\varphi_{1}\left(\widetilde{\varphi}^{\tau(t)-1}(x)\right)\right) \in \sigma_{1}(t-1)$. Второй вариант реализуется, если $\varphi_{1}\left(\widetilde{\varphi}^{\tau(t)-1}(x)\right) \notin \sigma_{2}(t-1)$ и $\widetilde{\varphi}^{\tau(t)}(x) \in\left\{x, \widetilde{\varphi}(x), \ldots, \widetilde{\varphi}^{\tau(t)-1}(x)\right\}$.

Аналогично предыдущему, если $\varphi_{1}, \varphi_{2}$ - случайные отображения, процесс $\mathcal{P}^{(2)}=\left\{\left(\sigma_{1}(t), \sigma_{2}(t), \nu(t)\right)\right\}_{t \geq 0}$ тоже является случайным с множеством состояний $2^{S} \times 2^{S} \times\{0,1, \ldots, N\}$. Начальным состоянием процесса является $\{(\varnothing, \varnothing, 0)\}$; состояния $(s, v, n) \in 2^{S} \times 2^{S} \times\{0,1, \ldots, N\}$ с $s=S$ - поглощающие. Проекция финального распределения процесса $\mathcal{P}^{(2)}$ на множестве 
$\{S\} \times 2^{S} \times\{1, \ldots, N\}$ на третий сомножитель $\{1, \ldots, N\}$ дает распределение числа компонент отображения $\widetilde{\varphi}$.

Если случайное отображение $\widetilde{\varphi}$ таково, что случайные образы $\varphi_{1}(x), \varphi_{2}(x), x \in S$, элементов независимы, и при любом $t=0,1, \ldots$ на $(t+1)$-м шаге начальный элемент $x \in S \backslash \sigma_{1}(t)$ выбирается независимо от значений отображений $\varphi_{1}, \varphi_{2}$ на множествах $\sigma_{1}(t), \sigma_{2}(t)$ соответственно, то при фиксированных $\sigma_{1}(t), \sigma_{2}(t)$ распределение последовательности $x, \varphi_{1}(x), \widetilde{\varphi}(x), \varphi_{1}(\widetilde{\varphi}(x)), \widetilde{\varphi}^{2}(x), \ldots, \widetilde{\varphi}^{\tau(t+1)}(x)$ (и, значит, последовательности $\left.\left\{\left(\sigma_{1}(s), \sigma_{2}(s), \nu(s)\right)\right\}_{s>t}\right)$ зависит только от $\sigma_{1}(t), \sigma_{2}(t), \nu(t)$ и не зависит от предыстории $\left\{\left(\sigma_{1}(s), \sigma_{2}(s), \nu(s)\right)\right\}_{0 \leq s<t}$; иными словами, для независимых отображений $\varphi_{1}, \varphi_{2}$ с независимыми образами элементов процесс $\mathcal{P}^{(2)}$ является марковским.

Если $\varphi_{1}, \varphi_{2}-$ независимые случайные равновероятные отображения, то для них образы элементов множества $S$ не только независимы, но и равномерно распределены на $S$. Поэтому марковский процесс $\mathcal{P}^{(2)}$ допускает укрупнение состояний: последовательность $\left\{\left(\left|\sigma_{1}(t)\right|,\left|\sigma_{2}(t)\right|, \nu(t)\right)\right\}_{t \geq 0}$ является цепью Маркова с дискретным временем и множеством состояний, содержащимся в $\{0,1, \ldots, N\}^{3}$. Число состояний укрупненной цепи Маркова существенно меньше, чем число $(N+1) 2^{2 N}$ состояний исходной цепи, и это позволяет находить финальное распределение, последовательно вычисляя распределения $\left\{\left(\left|\sigma_{1}(t)\right|,\left|\sigma_{2}(t)\right|, \nu(t)\right)\right\}, t=0,1, \ldots$, с помощью матрицы переходных вероятностей.

Выведем формулы для переходных вероятностей укрупненной цепи. Из состояния $\left(\left|\sigma_{1}(t)\right|,\left|\sigma_{2}(t)\right|, \nu(t)\right)=\left(m_{1}, m_{2}, v\right)$ укрупненная цепь может перейти только в состояния

$$
\begin{aligned}
\left(m_{1}+u, m_{2}+u, v\right), & \left(m_{1}+u, m_{2}+u-1, v\right), \\
\left(m_{1}+u, m_{2}+u, v+1\right), & \left(m_{1}+u, m_{2}+u-1, v+1\right),
\end{aligned}
$$

где $u \in\left\{1,2, \ldots, N-m_{1}\right\}$.

Переход $\left(m_{1}, m_{2}, v\right) \rightarrow\left(m_{1}+u, m_{2}+u, v\right)$ происходит, когда

$$
\begin{gathered}
\left(\left|\sigma_{1}(t)\right|,\left|\sigma_{2}(t)\right|, \nu(t)\right)=\left(m_{1}, m_{2}, v\right), \\
\varphi_{1}(x), \varphi_{1}(\widetilde{\varphi}(x)), \ldots, \varphi_{1}\left(\widetilde{\varphi}^{u-1}(x)\right) \notin \sigma_{2}(t) \text { попарно различны, } \\
x, \varphi_{2}\left(\varphi_{1}(x)\right), \varphi_{2}\left(\varphi_{1}(\widetilde{\varphi}(x))\right), \ldots, \varphi_{2}\left(\varphi_{1}\left(\widetilde{\varphi}^{u-2}(x)\right)\right) \notin \sigma_{1}(t) \text { попарно различны }
\end{gathered}
$$

$$
\varphi_{2}\left(\varphi_{1}\left(\widetilde{\varphi}^{u-1}(x)\right)\right)=\widetilde{\varphi}^{u}(x) \in \sigma_{1}(t) ;
$$


поэтому

$$
\begin{gathered}
p_{\left(m_{1}, m_{2}, v\right),\left(m_{1}+u, m_{2}+u, v\right)}= \\
=\frac{m_{1}}{N}\left(1-\frac{m_{2}}{N}\right) \prod_{k=1}^{u-1}\left(1-\frac{m_{1}+k}{N}\right)\left(1-\frac{m_{2}+k}{N}\right) .
\end{gathered}
$$

Переход $\left(m_{1}, m_{2}, v\right) \rightarrow\left(m_{1}+u, m_{2}+u-1, v\right)$ происходит, когда

$$
\begin{gathered}
\left(\left|\sigma_{1}(t)\right|,\left|\sigma_{2}(t)\right|, \nu(t)\right)=\left(m_{1}, m_{2}, v\right), \\
\varphi_{1}\left(\widetilde{\varphi}^{u-1}(x)\right) \in \sigma_{2}(t),
\end{gathered}
$$

и при $u>1$

$$
\varphi_{1}(x), \varphi_{1}(\widetilde{\varphi}(x)), \ldots, \varphi_{1}\left(\widetilde{\varphi}^{u-2}(x)\right) \notin \sigma_{2}(t) \text { попарно различны, }
$$

$x, \varphi_{2}\left(\varphi_{1}(x)\right), \varphi_{2}\left(\varphi_{1}(\widetilde{\varphi}(x))\right), \ldots, \varphi_{2}\left(\varphi_{1}\left(\widetilde{\varphi}^{u-2}(x)\right)\right) \notin \sigma_{1}(t)$ попарно различны; поэтому

$$
\begin{gathered}
p_{\left(m_{1}, m_{2}, v\right),\left(m_{1}+u, m_{2}+u-1, v\right)}= \\
=\frac{m_{2}}{N} \prod_{k=1}^{u-1}\left(1-\frac{m_{1}+k}{N}\right)\left(1-\frac{m_{2}+k-1}{N}\right) .
\end{gathered}
$$

Переход $\left(m_{1}, m_{2}, v\right) \rightarrow\left(m_{1}+u, m_{2}+u, v+1\right)$ происходит, когда

$$
\left(\left|\sigma_{1}(t)\right|,\left|\sigma_{2}(t)\right|, \nu(t)\right)=\left(m_{1}, m_{2}, v\right),
$$

$\varphi_{1}(x), \varphi_{1}(\widetilde{\varphi}(x)), \ldots, \varphi_{1}\left(\widetilde{\varphi}^{u-1}(x)\right) \notin \sigma_{2}(t)$ попарно различны,

$x, \varphi_{2}\left(\varphi_{1}(x)\right), \varphi_{2}\left(\varphi_{1}(\widetilde{\varphi}(x))\right), \ldots, \varphi_{2}\left(\varphi_{1}\left(\widetilde{\varphi}^{u-2}(x)\right)\right) \notin \sigma_{1}(t)$ попарно различны

и

$$
\widetilde{\varphi}^{u}(x) \in\left\{x, \tilde{\varphi}(x), \ldots, \widetilde{\varphi}^{u-1}(x)\right\}
$$

поэтому

$$
\begin{gathered}
p_{\left(m_{1}, m_{2}, v\right),\left(m_{1}+u, m_{2}+u, v+1\right)}= \\
=\frac{u}{N}\left(1-\frac{m_{2}}{N}\right) \prod_{k=1}^{u-1}\left(1-\frac{m_{1}+k}{N}\right)\left(1-\frac{m_{2}+k}{N}\right) .
\end{gathered}
$$

Переход $\left(m_{1}, m_{2}, v\right) \rightarrow\left(m_{1}+u, m_{2}+u-1, v+1\right)$ происходит, когда

$$
\begin{gathered}
\left(\left|\sigma_{1}(t)\right|,\left|\sigma_{2}(t)\right|, \nu(t)\right)=\left(m_{1}, m_{2}, v\right), \\
\varphi_{1}\left(\widetilde{\varphi}^{u-1}(x)\right) \in \sigma_{2}(t),
\end{gathered}
$$


и при $u>1$

$$
\begin{gathered}
\varphi_{1}(x), \varphi_{1}(\widetilde{\varphi}(x)), \ldots, \varphi_{1}\left(\widetilde{\varphi}^{u-2}(x)\right) \notin \sigma_{2}(t) \text { попарно различны, } \\
x, \varphi_{2}\left(\varphi_{1}(x)\right), \varphi_{2}\left(\varphi_{1}(\widetilde{\varphi}(x))\right), \ldots, \varphi_{2}\left(\varphi_{1}\left(\widetilde{\varphi}^{u-2}(x)\right)\right) \notin \sigma_{1}(t) \text { попарно различны; }
\end{gathered}
$$

поэтому

$$
\begin{gathered}
p_{\left(m_{1}, m_{2}, v\right),\left(m_{1}+u, m_{2}+u-1, v+1\right)}= \\
=\frac{u-1}{N} \prod_{k=1}^{u-1}\left(1-\frac{m_{1}+k}{N}\right)\left(1-\frac{m_{2}+k-1}{N}\right) .
\end{gathered}
$$

Таким образом, если $q^{(2)}\left(m_{1}, m_{2}, u\right)=\prod_{k=1}^{u-1}\left(1-\frac{m_{1}+k}{N}\right)\left(1-\frac{m_{2}+k}{N}\right)$, то в силу (6)-(9)

$$
p_{(m, n, v),(m+u, n+u-r, v+s)}= \begin{cases}\frac{m}{N}\left(1-\frac{n}{N}\right) q^{(2)}(m, n, u), & r=0, s=0, \\ \frac{n}{N} q^{(2)}(m, n-1, u), & r=1, s=0, \\ \frac{u}{N}\left(1-\frac{n}{N}\right) q^{(2)}(m, n, u), & r=0, s=1, \\ \frac{u-1}{N} q^{(2)}(m, n-1, u), & r=1, s=1 .\end{cases}
$$

Значения $p_{(m, n, v),(m+u, n+u-r, v+s)}$ можно вычислить заранее. Множество состояний укрупненной цепи состоит из $O\left(N^{3}\right)$ элементов; ввиду того, что в каждой строке матрицы вероятностей переходов отличны от 0 не более $4 \mathrm{~N}$ элементов, вычисление изменения распределения цепи за один шаг можно провести за $O\left(N^{4}\right)$ арифметических операций, а если объединить все состояния с $v>M$ в одно поглощающее состояние - то за $O\left(M N^{3}\right)$ операций. Максимальное число шагов укрупненной цепи до попадания в множество финальных состояний не превышает $N$.

В Таблице 2 жирным шрифтом выделены распределения $\boldsymbol{P}\left\{\nu_{N}=v\right\}$ чисел компонент для итерации двух равновероятных отображений, а светлым шрифтом, для сравнения, - распределения чисел компонент для равновероятных отображений. Видно, что при переходе от равновероятных отображений к их итерациям распределение чисел компонент смещается к 0.

Предложенные выше алгоритмы легко модифицировать, например, для вычисления распределений числа циклических точек $\lambda_{N}$ случайного отображения множества $S=\{1, \ldots, N\}$ в себя. Для этого достаточно заменить в процессах последовательного определения отображений последнюю компоненту, соответствующую числу связных компонент, суммой длин циклов уже образовавшихся компонент. 
Таблица 2. Распределения $\boldsymbol{P}\left\{\nu_{N}=v\right\}$ чисел компонент итераций двух случайных равновероятных отображений и обычных случайных равновероятных отображений

\begin{tabular}{|c|c|c|c|}
\hline$\vartheta$ & $N=100$ & $N=250$ & $N=500$ \\
\hline $\mathbf{1}$ & $\mathbf{0 . 1 6 9 0 8 6 8}$ & $\mathbf{0 . 1 0 8 8 1 1 6}$ & $\mathbf{0 . 0 7 7 6 1 6 0}$ \\
равновер. & 0.1220996 & 0.0779592 & 0.0553925 \\
\hline $\mathbf{2}$ & $\mathbf{0 . 3 2 3 6 2 4 9}$ & $\mathbf{0 . 2 5 7 4 3 7 7}$ & $\mathbf{0 . 2 1 0 3 0 1 2}$ \\
равновер. & 0.2750239 & 0.2110974 & 0.1691089 \\
\hline $\mathbf{3}$ & $\mathbf{0 . 2 8 1 5 1 1 4}$ & $\mathbf{0 . 2 8 4 8 8 3 3}$ & $\mathbf{0 . 2 7 0 3 3 0 7}$ \\
равновер. & 0.2874088 & 0.2708997 & 0.2473149 \\
\hline $\mathbf{4}$ & $\mathbf{0 . 1 5 0 2 7 8 2}$ & $\mathbf{0 . 1 9 8 3 6 4 3}$ & $\mathbf{0 . 2 2 1 3 2 7 6}$ \\
равновер. & 0.1876120 & 0.2212013 & 0.2322667 \\
\hline $\mathbf{5}$ & $\mathbf{0 . 0 5 5 8 7 9 7}$ & $\mathbf{0 . 0 9 8 4 0 7 1}$ & $\mathbf{0 . 1 3 0 5 0 6 3}$ \\
равновер. & 0.8666421 & 0.1299575 & 0.1582343 \\
\hline $\mathbf{6}$ & $\mathbf{0 . 0 1 5 5 4 0 2}$ & $\mathbf{0 . 0 3 7 2 8 4 0}$ & $\mathbf{0 . 0 5 9 3 5 0 2}$ \\
равновер. & 0.0303775 & 0.0588295 & 0.0836767 \\
\hline $\mathbf{7}$ & $\mathbf{0 . 0 0 3 3 8 4 0}$ & $\mathbf{0 . 0 1 1 2 8 1 4}$ & $\mathbf{0 . 0 2 1 7 5 1 8}$ \\
равновер. & 0.0084510 & 0.0214419 & 0.0358706 \\
\hline $\mathbf{8}$ & $\mathbf{0 . 0 0 0 5 9 5 9}$ & $\mathbf{0 . 0 0 2 8 1 2 8}$ & $\mathbf{0 . 0 0 6 6 2 5 2}$ \\
равновер. & 0.0019256 & 0.0064886 & 0.0128485 \\
\hline $\mathbf{9}$ & $\mathbf{0 . 0 0 0 0 8 6 9}$ & $\mathbf{0 . 0 0 0 2 8 1 3}$ & $\mathbf{0 . 0 0 1 7 1 5 5}$ \\
равновер. & 0.0003678 & 0.0016677 & 0.0039324 \\
\hline $\mathbf{1 0}$ & $\mathbf{0 . 0 0 0 0 1 0 7}$ & $\mathbf{0 . 0 0 0 1 0 6 8}$ & $\mathbf{0 . 0 0 0 3 8 4 3}$ \\
равновер. & 0.0000600 & 0.0003705 & 0.0010463 \\
\hline $\mathbf{1 1}$ & $\mathbf{0 . 0 0 0 0 0 1 1}$ & $\mathbf{0 . 0 0 0 0 1 6 8}$ & $\mathbf{0 . 0 0 0 0 7 5 5}$ \\
равновер. & 0.0000085 & 0.0000722 & 0.0002453 \\
\hline $\mathbf{1 2}$ & $\mathbf{0 . 0 0 0 0 0 0 1}$ & $\mathbf{0 . 0 0 0 0 0 2 3}$ & $\mathbf{0 . 0 0 0 0 1 3 2}$ \\
равновер. & 0.0000010 & 0.0000125 & 0.0000513 \\
\hline & & &
\end{tabular}

В случае равновероятных отображений точная формула для распределения $\lambda_{N}$ приведена в [5], см. также [1]:

$$
\boldsymbol{P}\left\{\lambda_{N}=n\right\}=\frac{n(N-1) !}{N^{n}(N-n) !} .
$$

Известно также предельное распределение числа циклических точек:

$$
\lim _{n \rightarrow \infty} \boldsymbol{P}\left\{N^{-1 / 2} \lambda_{N} \geq z\right\}=e^{-z^{2} / 2} .
$$


Мы опишем переходные вероятности цепей Маркова для равновероятных отображений и итераций двух равновероятных отображений, чтобы выявить их различия.

Если $\varphi: S \rightarrow S-$ равновероятное отображение, то при условии, что на $t$-м шаге образуется новая связная компонента размера $u$ (т. е. при условии $\left.\min \left\{k: \varphi^{k}(x) \in\left\{x, \varphi(x), \ldots, \varphi^{k-1}(x)\right\}\right\}=u\right)$, длина ее цикла имеет равномерное распределение на множестве $\{1, \ldots, u\}$. Поэтому формулы для переходных вероятностей цепи Маркова $\{(|\sigma(t)|, \lambda(t))\}_{t \geq 0}$, где $|\sigma(t)|-$ число элементов множества $\sigma(t)$ тех $x \in S$, для которых значение $\varphi(x)$ было определено на каком-нибудь из первых $t$ шагов, а $\lambda(t)$ - число циклических точек в $\sigma(t)$, имеют следующий вид:

$$
\begin{gathered}
p_{(m, v),(m+u, v)}=\frac{m}{N} q(m, u), \quad p_{(m, v),(m+u, v+j)}=\frac{1}{N} q(m, u), \quad j=1, \ldots, u, \\
q(m, u)=\prod_{k=1}^{u-1}\left(1-\frac{m+k}{N}\right) .
\end{gathered}
$$

Если случайное отображение $\widetilde{\varphi}(x)=\varphi_{2}\left(\varphi_{1}(x)\right)$ строится как итерация двух независимых равновероятных отображений $\varphi_{1}, \varphi_{2}: S \rightarrow S$, то следует рассматривать цепь Маркова $\left\{\left(\left|\sigma_{1}(t)\right|,\left|\sigma_{2}(t)\right|, \lambda(t)\right)\right\}_{t \geq 0}$, где $\sigma_{j}(t) \subset S-$ множество всех элементов $x \in S$, для которых значение $\varphi_{j}(x), j=1,2$, было определено на каком-нибудь из первых $t$ шагов, а $\lambda(t)$ - число циклических точек в $\sigma_{1}(t)$. При условии, что на $t$-м шаге образуется новая связная компонента размера $u$ (т.е. при условии $\left|\sigma_{1}(t)\right|-\left|\sigma_{1}(t-1)\right|=$ $\left.=\min \left\{k: \widetilde{\varphi}^{k}(x) \in\left\{x, \widetilde{\varphi}(x), \ldots, \widetilde{\varphi}^{k-1}(x)\right\}\right\}=u\right)$, длина ее цикла $\lambda(t)$ имеет распределение, немного отличающееся от равномерного распределения на множестве $\{1, \ldots, u\}$ :

$$
\boldsymbol{P}\left\{\lambda(t)=s|| \sigma_{1}(t)|-| \sigma_{1}(t-1) \mid=u\right\}= \begin{cases}\frac{2-\frac{u-1}{N}}{2 u-1-u \frac{u-1}{N}}, & s=1, \ldots, u-1, \\ \frac{1-\frac{u-1}{N}}{2 u-1-u \frac{u-1}{N}}, & s=u,\end{cases}
$$

так как

$$
\begin{gathered}
\boldsymbol{P}\left\{\widetilde{\varphi}^{u}(x)=\widetilde{\varphi}^{u-s}(x)\right\}=\boldsymbol{P}\left\{\varphi_{1}\left(\widetilde{\varphi}^{u-1}(x)\right)=\varphi_{1}\left(\widetilde{\varphi}^{u-s-1}(x)\right)\right\}+ \\
+\boldsymbol{P}\left\{\varphi_{1}\left(\widetilde{\varphi}^{u-1}(x)\right) \notin\left\{\varphi_{1}(x), \ldots, \varphi_{1}\left(\widetilde{\varphi}^{u-2}(x)\right)\right\}, \varphi_{2}\left(\varphi_{1}\left(\widetilde{\varphi}^{u-1}(x)\right)\right)=\widetilde{\varphi}^{u-s}(x)\right\}= \\
= \begin{cases}\frac{1}{N}\left(2-\frac{u-1}{N}\right), & s=1, \ldots, u-1, \\
\frac{1}{N}\left(1-\frac{u-1}{N}\right), & s=u .\end{cases}
\end{gathered}
$$


Поэтому переходные вероятности цепи Маркова $\left\{\left(\left|\sigma_{1}(t)\right|,\left|\sigma_{2}(t)\right|, \lambda(t)\right)\right\}_{t \geq 0}$ имеют вид

$$
p_{(m, n, v),(m+u, n+u-r, v+s)}= \begin{cases}\frac{m}{N}\left(1-\frac{n}{N}\right) q^{(2)}(m, n, u), & r=0, s=0 \\ \frac{n}{N} q^{(2)}(m, n-1, u), & r=1, s=0 \\ \frac{1}{N}\left(1-\frac{n}{N}\right) q^{(2)}(m, n, u), & r=0, s=1, \ldots, u \\ \frac{1}{N} q^{(2)}(m, n-1, u), & r=1, s=1, \ldots, u-1\end{cases}
$$

Таблица 3. Функции распределения $\boldsymbol{P}\left\{\lambda_{N} \leq v\right\}$ чисел циклических точек случайных равновероятных отображений и итераций двух равновероятных отображений

\begin{tabular}{|c|c|c|c|c|}
\hline$v$ & отображение & $N=100$ & $N=225$ & $N=400$ \\
\hline $\mathbf{1}$ & равновер. & 0.0100000 & 0.0044444 & 0.0025000 \\
& итерация & 0.0199000 & 0.0088691 & 0.0049937 \\
\hline $\mathbf{5}$ & равновер. & 0.1417223 & 0.0650073 & 0.0369723 \\
& итерация & 0.2633593 & 0.1257887 & 0.0725776 \\
\hline $\mathbf{1 0}$ & равновер. & 0.4346591 & 0.2199038 & 0.1295275 \\
& итерация & 0.6803897 & 0.3914499 & 0.2422777 \\
\hline $\mathbf{1 5}$ & равновер. & 0.7184084 & 0.4207493 & 0.2621036 \\
& итерация & 0.9207062 & 0.6644686 & 0.4555088 \\
\hline $\mathbf{2 0}$ & равновер. & 0.8956804 & 0.6182711 & 0.4138654 \\
& итерация & 0.9891174 & 0.8542830 & 0.6564462 \\
\hline $\mathbf{2 5}$ & равновер. & 0.9717867 & 0.7773893 & 0.5640980 \\
& итерация & 0.9992040 & 0.9504445 & 0.8099894 \\
\hline $\mathbf{3 0}$ & равновер. & 0.9945462 & 0.8854728 & 0.6967590 \\
& итерация & 0.9999703 & 0.9868835 & 0.9080449 \\
\hline $\mathbf{3 5}$ & равновер. & 0.9992645 & 0.9481865 & 0.8028475 \\
& итерация & 0.9999995 & 0.9973154 & 0.9611309 \\
\hline $\mathbf{4 0}$ & равновер. & 0.9999327 & 0.9794564 & 0.8803181 \\
& итерация & 0.9999999 & 0.9995780 & 0.9856762 \\
\hline $\mathbf{4 5}$ & равновер. & 1.0000000 & 0.9928870 & 0.9322276 \\
& итерация & 1.0000000 & 0.9999494 & 0.9954069 \\
\hline $\mathbf{5 0}$ & равновер. & 1.0000000 & 0.9978574 & 0.9642358 \\
& итерация & 1.0000000 & 0.9999954 & 0.9987209 \\
\hline $\mathbf{5 5}$ & равновер. & 1.0000000 & 0.9994408 & 0.9824298 \\
& итерация & 1.0000000 & 0.9999997 & 0.9996913 \\
\hline \multirow{20}{*}{ матем.ож. } & равновер. & 1.0000000 & 0.9998741 & 0.9919723 \\
& итерация & 1.0000000 & 1.0000000 & 0.9999355 \\
\hline \multirow{2}{*}{ равновер. } & 12.2089414 & 18.4715471 & 24.7240632 \\
& итерация & 8.7158770 & 13.1402552 & 17.5680100 \\
\hline & равновер. & 38.6866151 & 90.2149091 & 162.790770 \\
& итерация & 19.9319649 & 46.0176380 & 82.8337996 \\
\hline
\end{tabular}


Таблица 3 содержит значения вычисленных по формулам (13) и (11) функций распределения и первых двух моментов числа $\lambda_{N}$ циклических точек равновероятного отображения, а также вычисленных по формулам (14) функций распределения и первых двух моментов числа циклических точек итерации двух равновероятных отображений для $N=100,225,400$. Видно, что в обоих случаях число циклических точек растет приблизительно пропорционально $\sqrt{N}$, но (судя по моментам) коэффициент пропорциональности для итерации двух отображений примерно в $\sqrt{2}$ раз меньше, чем для равновероятных отображений.

\section{Список литературы}

1. Колчин В. Ф. Один класс предельных теорем для условных распределений. Лит. матем. сб., 1968, т. 8, № 1, с. 53-63.

2. Колчин В. Ф. Случайные отображения. - М.: Наука, 1984.

3. Flajolet P., Odlyzko A.M. Random mapping statistics. - Lect. Notes Comp. Sci., 1989, v. 434, p. 329-354.

4. Harris B. A survey of the early history of the theory of random mappings. - In: Probabilistic Methods in Discrete Mathematics. - Moscow: TVP-VSP, 1993, p. 1-22.

5. Harris $B$. Probability distributions related to random mappings. - Ann. Math. Statist., 1960, v. 31, № 4, p. 1045-1062. 